\title{
Comparison of fission induced by nucleons and pions
}

\author{
Zafar Yasin ${ }^{1,5}$, R.J. Peterson ${ }^{2}$, M. Ikram Shahzad ${ }^{3}$, and H.A. Khan ${ }^{4}$ \\ 1 Department of Nuclear Engineering, PIEAS, P.O. Nilore, Islamabad, Pakistan \\ 2 Department of Physics, University of Colorado, Boulder, CO 80309-0390, USA \\ 3 Physics Research Division, PINSTECH, P.O. Nilore, Islamabad, Pakistan \\ 4 COMSATS, Shahrah-e-Jamhuriyyat, G-5/2, Islamabad, Pakistan \\ 5 Max-Lab, Lund University, Lund 21 00, Sweden
}

\begin{abstract}
The goal of the present work is to compare properties of fission induced by nucleons and pions. Three different methodologies are used to make this comparison. Firstly, it is shown that pion-induced fission cross sections are not much different in magnitude from proton induced fission cross sections at the same kinetic energy. Secondly, across the pion-nucleon $(3,3)$ resonance so prominent with free nucleons, it is observed that there is no significant change in the reaction mechanism in the case of pions. Thirdly, it is noted that an empirical expression used for evaluations of proton induced fission cross sections is also valid for positive pion-induced fission cross sections, at least for actinide nuclei.
\end{abstract}

Nuclear fission is an important, interesting and complex nuclear reaction, with relevance in areas ranging from nuclear structure models to accelerator-driven systems (ADS). There is need in basic and applied nuclear physics to understand the properties of fission induced by nucleons and pions, and cross sections for fission induced by nucleons and pions have applications in advanced nuclear reactor technologies, in medicine, for effects on electronics, etc. In ADS using high energy protons, pions are created from the primary proton reactions, and are then part of complex cascades of reactions. Hence, fission induced by nucleons and pions are both important, and fission reactions may have a significant effect on spallation target performance and may contribute to the production of radioactive or chemically hazardous materials in targets [1].

Fission induced by pions is not as much studied as fission induced by nucleons. Hence, the goal of the present work is to compare properties of fission induced by nucleons and pions. Three different methodologies are used to make this comparison. Firstly, it is shown that pion-induced fission cross sections are not much different in magnitude from proton induced fission cross sections at the same beam kinetic energy. Secondly, across the pion-nucleon $(3,3)$ resonance so prominent with free nucleons, it is observed that there is no significant change in the reaction mechanism in the case of pions. Thirdly, it is noted that an empirical expression used for evaluations of proton induced fission cross sections is also valid for positive pion-induced fission cross sections, at least for actinide nuclei. Fission cross sections computed using a cascade-exciton model code for ${ }^{209} \mathrm{Bi}$ and ${ }^{238} \mathrm{U}$ are also compared with available experimental data.

Proton and pion induced fission cross sections are computed using the cascade-exciton model code CEM95. Three stages are incorporated in CEM95; cascade, preequilibrim and compound nucleus stages. A change in the ratio of the level density parameter for fission and neutron emission channels $\left(\mathrm{a}_{f} / \mathrm{a}_{n}\right)$ is taken into account with change in the incident energy of the projectile to account for the change in the excitation energy of the compound nucleus. Other parameters are the same as are used in our paper [2]. A statistical weight method is used to compute the cross sections for fission. According to this method the fission cross sections are estimated as

$$
\sigma_{f}=\frac{\sigma_{i n}}{N_{i n}} \sum_{i=1}^{N_{i n}}\left(W_{f}\right)_{i},
$$

where $W_{f}$ is the probability for the nucleus to fission at any of the chain stages, $N_{i n}$ is the total number of the cascades followed and $\sigma_{i n}$ is the total inelastic cross section.

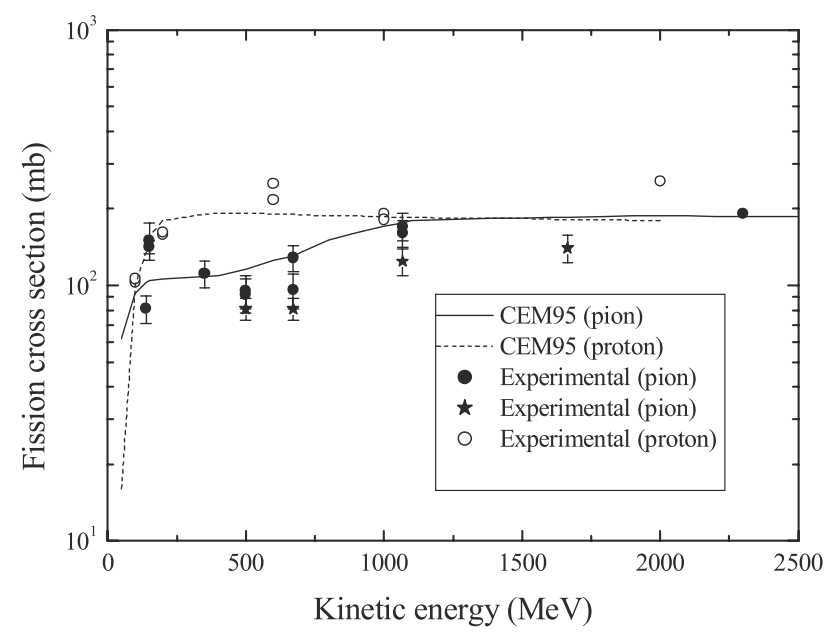

Fig. 1. Fission induced by negative pions is compared with the fission induced by protons for ${ }^{209} \mathrm{Bi}$. The solid curve indicates the cross sections computed using CEM95 for negative pion induced fission in ${ }^{209} \mathrm{Bi}$ and dashed curve for proton induced fission in ${ }^{209} \mathrm{Bi}$. Solid circles are the data for negative pion induced fission from ref. [3], star from ref. [4] and open circles are data from refs. [5-10] for proton induced fission.

Cross sections for fission induced by negative pions are compared with the cross sections for fission induced by protons in figures 1 and 2 for ${ }^{209} \mathrm{Bi}$ and ${ }^{238} \mathrm{U}$, respectively. The 


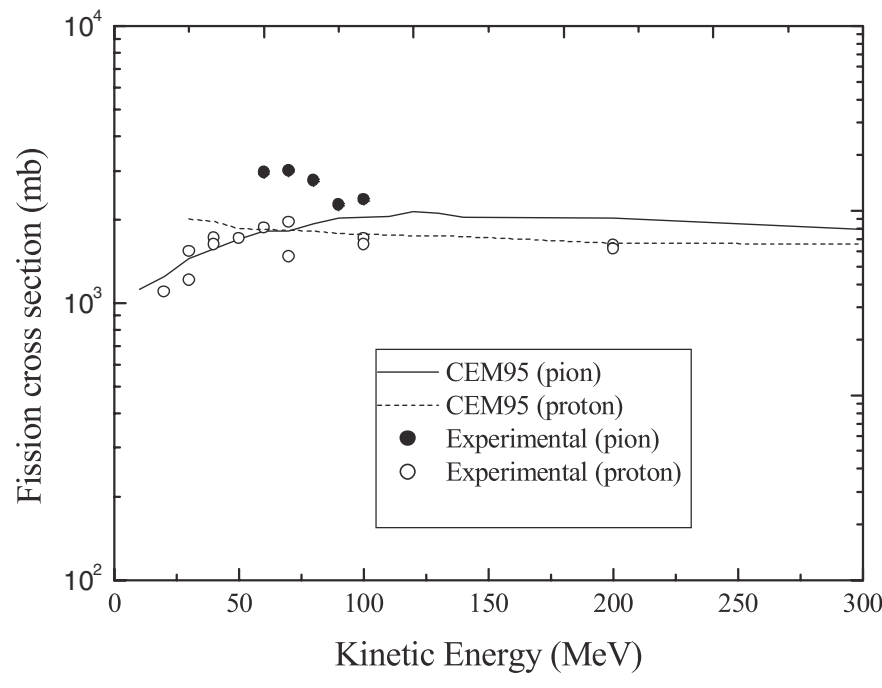

Fig. 2. As figure 1, but for ${ }^{238} \mathrm{U}$. Solid circles show the experimental data for negative pion induced fission from ref. [11] whereas open circles are the experimental data from refs. $[9,10,12-15]$ for proton induced fission.

ratio of the level density parameter used lies in the range 1.191.06 for negative pion fission in ${ }^{209} \mathrm{Bi}, 1.21-1.058$ for proton induced fission in ${ }^{209} \mathrm{Bi}$ and is 1.17 for ${ }^{238} \mathrm{U}$. It can be seen that negative pion induced fission cross sections are not much different in magnitude from the proton induced fission cross sections. For ${ }^{209} \mathrm{Bi}$, below $1 \mathrm{GeV}$ the differences observed between pion and proton induced fission are reported by the calculations with CEM95.

To compare pion induced fission with nucleon induced fission, an empirical analysis for positive pion induced fission cross sections is performed, based on the well known expression that has been used for proton induced fission cross sections [1]. The pion cross sections are taken to be given by

$$
\sigma_{f}\left(E_{p}\right)=P_{1}\left\{1-\exp \left[-P_{3}\left(E_{p}-P_{2}\right)\right]\right\} \times\left(1-P_{4} \ln E_{p}\right),
$$

where $\sigma_{f}$ is the fission cross section ( $\left.\mathrm{mb}\right), \mathrm{E}_{\mathrm{p}}$ is the incident proton or pion kinetic energy $(\mathrm{MeV})$ and $\mathrm{P}_{1}, \mathrm{P}_{2}, \mathrm{P}_{3}$, are fitting parameters known as the saturation cross section, the apparent threshold-energy and the increasing rate, respectively. $\mathrm{P}_{4}$ is the parameter introduced [1] in order to reproduce the decrease of fission cross sections at high energies. The parameters $\mathrm{P}_{i}$ for protons have been determined using equation (5) of ref. [1] for $\mathrm{Bi}$ and $\mathrm{P}_{1}$ for ${ }^{238} \mathrm{U}$ is determined from equation (6) of ref. [1]; other parameters are taken from table 3 of ref. [1]. All of these are deduced from proton-induced fission, and are here used to compute the pion cross sections shown below.

Calculated cross sections for fission induced by positive pions in ${ }^{209} \mathrm{Bi}$ and ${ }^{238} \mathrm{U}$ are shown in figures 3 and 4 , respectively, along with use of the proton parameters in the empirical expression above. The cross sections are also compared with the experimental data. The ratio of the level density parameter used in CEM lies in the range 1.19-1.057 for ${ }^{209} \mathrm{Bi}$ and $1.19-1.07$ for ${ }^{238} \mathrm{U}$. In the case of ${ }^{209} \mathrm{Bi}$, there is pronounced disagreement between the pion data and the expectation from proton parameters at lower energies, but there is good agreement at higher energies. At low energies,

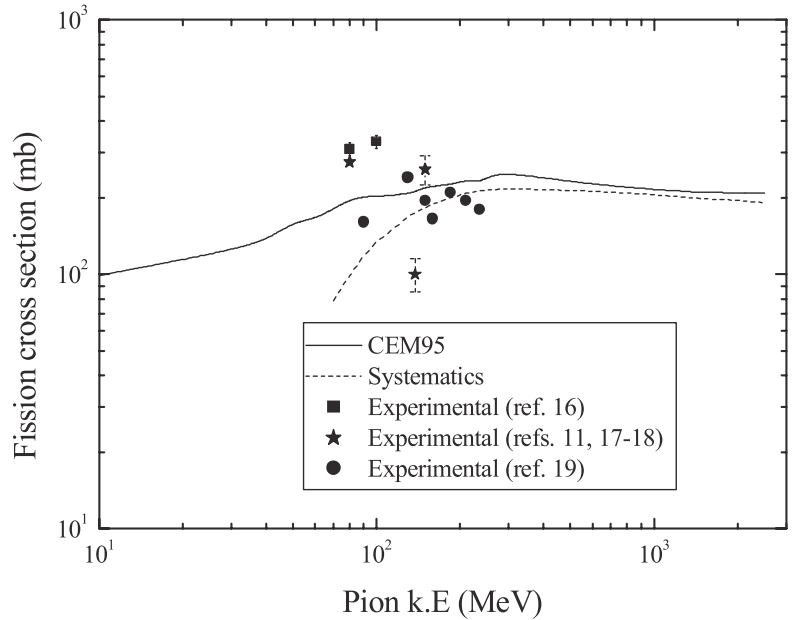

Fig. 3. Fission cross sections induced by positive pions in ${ }^{209} \mathrm{Bi}$ from the present CEM calculations and from use of the empirical expression (2) found for protons are shown as solid and dotted curves, respectively. Fission cross sections are compared with the experimental data, shown as solid squares, solid circles and stars from refs. [11,16-19], respectively.

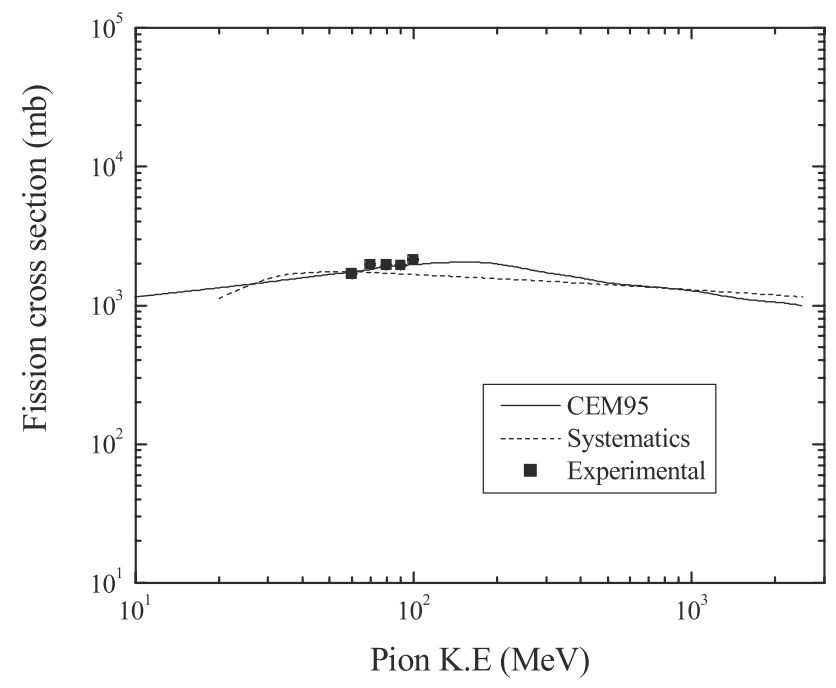

Fig. 4. As figure 3, but for ${ }^{238} \mathrm{U}$. Fission cross sections are compared with the experimental data as solid squares, from ref. [11].

the pion "true absorption" mechanism may be important, but not possible for protons. For ${ }^{238} \mathrm{U}$ there is good matching of the cross sections.

Recently $[2,19]$ fission cross sections and fission probabilities have been calculated for positive and negative pion induced fission of different heavy nuclei in small steps across the $(3,3)$ resonance to observe any possible resonant effects in complex nuclei across this sensitive region. The lack of any pronounced resonant peak indicates that there is no significant change in the reaction mechanism in this important energy range.

On the basis of the three different methodologies: magnitudes of fission cross sections, use of an empirical relation analysis, and fission across the $(3,3)$ resonance, we can conclude that pion induced fission is similar to nucleon induced fission, except at low pion energies. This is due to the fact 
that the excitation energy imparted to the compound nucleus is much the same, with no apparent role of the pion rest mass of $140 \mathrm{MeV}$.

I thank Prof. Dr Bent Schroder for helping me to improve this manuscript and for giving me a chance to work under his kind supervision at Max-Lab. We thank COMSATS, Pakistan, and MaxLab, Sweden, for the traveling grant.

\section{References}

1. A.V. Prokofiev, Nucl. Instrum. Meth. A 463, 557 (2001).

2. Z. Yasin, M. Ikram Shahzad, Nucl. Phys. A 773, 221 (2006).

3. Z. Yasin et al., Nucl. Phys. A 765, 390 (2006).

4. H.A. Khan et al., Rad. Meas. 28, 287 (1997).

5. B.A. Bochagov et al., Sov. J. Nucl. Phys. 28, 291 (1978).
6. M. Debeauvais et al., in Proceedings of the Ninth International Conference on Solid State Nuclear Track Detectors, 1976, Neuherberg, Vol. 2 (1978), p. 1179.

7. J. Huids, S. Katcoff, Phys. Rev. C 13, 1961 (1976).

8. J. Huids, S. Katcoff, Phys. Rev. 180, 1122 (1969).

9. V.S. Bychenkov et al., Yad. Fiz. 17, 947 (1973).

10. O.E. Shigaev et al., Preprint RI-17, 1973 (in Russian).

11. K.H. Hicks et al., Phys. Rev. C 31, 1323 (1985).

12. J.R. Boyce et al., Phys. Rev. C 10, 231 (1974).

13. P.C. Stevenson et al., Phys. Rev. 111, 886 (1958).

14. S. Baba et al., Nucl. Phys. A 175, 177 (1971).

15. A.N. Smirnov et al., 21 Int. Symp. on Nucl. Phys., Gaussing, 1991, p. 214.

16. R.J. Peterson et al., Z. Phys. A 352, 181 (1995).

17. S. de Barros et al., Nucl. Phys. A 542, 511 (1992).

18. S. de Barros et al., Z. Phys. A 359, 35 (1997).

19. R.J. Peterson et al., Eur. Phys. J. A 10, 69 (2001). 